\title{
Neuropéptide Y, anti-mullerian and reproductive hormones and their relationship with vasomotor symptoms in menopausal transition
}

\begin{abstract}
Objetive: Determine the association between concentrations of anti-Mullerian hormone, neuropeptide $\mathrm{Y}$, follicle stimulating hormone and estrogens with vasomotor symptoms in peri- and postmenopausal women.

Methods: Healthy peri- and postmenopausal women aged 40-60 years were invited to participate in this study. A total of 110 women were recruited and completed the baseline questionnaire to divide into peri- and postmenopausal women and Hamilton Belch Raphaelsen and vasomotor symptomatology questionnaires. Glucose, lipid profile, NPY, FSH, E2 y AMH in serum was evaluated.

Results: A total of 108 women completed the study. A 57\% were perimenopausial (group 1; 45.8 \pm 3.1 years) and $42 \%$ postmenopausial (group 2; $51.6 \pm 3.9$ years).FSH, IMC and sleep disturbances were an independent predictor of the duration of hot flashes $(\mathrm{P}<0.001)$. NPY concentrations were associated with HDL, schooling and number of births $(\mathrm{R} 2=0.12, \mathrm{P}<0.01, \mathrm{P}<0.003, \mathrm{P}<0.03)$. HDL was an independent predictor of AMR $(\mathrm{R} 2=0.03,<0.04)$. The levels of NPY and FSH were higher in group 2 and the levels of AMH and E2 in group 1.
\end{abstract}

Conclusions: There is an association between duration of hot flashes with sleep disorders, levels of FSH and BMI. The levels of AMH correlated with low levels of HDL.

Keywords : neuropeptide Y, anti-müllerian hormone, perimenopause, postmenopause, hot flashes, sleep disturbances, follicle stimulating hormone
Volume 9 Issue 4 - 2018

\author{
Aguilera-Elizarraraz NL,' Franco-Robles E,' \\ Hernández-Marín JA,' Malacara-Hernándezb \\ $\mathrm{JM}^{2}$ \\ 'Life Sciences Division, Campus Irapuato-Salamanca, University \\ of Guanajuato, Mexico \\ ${ }^{2}$ Department of Medical Sciences, Division of Health Sciences, \\ University of Guanajuato, Mexico
}

\begin{abstract}
Correspondence: IDr. Norma Lilia Aguilera Elizarraraz, Department of Nursing and Obstetrics, Division of Life Sciences, Campus Irapuato-Salamanca, University of Guanajuato. Exhaust the Copal km 9, Irapuato-Silao highway, C.P. 36824, Apartado Postal 3II, Irapuato, Guanajuato, Mexico, Email normaaguilerae@hotmail.com
\end{abstract}

Received: July 09, 2018| Published: August 06, 2018

\section{Introduction}

Menopause occurs at the end of ovarian function and is diagnosed after 12 months of amenorrhea in women who have had regular menstrual cycles. Premenopause ends with perimenopause because the woman presents symptoms of menopause. Hormonal trends can be found in menopause changes; therefore, it is also called transition to menopause (TM). The TM begins with the variation in the duration of the menstrual cycle caused by the elevation in concentrations of follicle stimulating hormone (FSH) and ends with the final menstrual period. Post-menopause comprises from the last menstrual period to the end in the life of women and is divided into early and late stages. ${ }^{1}$ During menopause, the levels of estrogens and androgens decrease, which is associated with several signs and symptoms, such as loss of bone mineral density, vasomotor symptoms, reduced sexual interest, mood changes, loss of energy, among others. ${ }^{2}$

In this transition, the hormonal concentrations that undergo major changes are FSH, anti-Müllerian hormone (AMH), inhibin B and estradiol (E2). AMH appears to be the first marker of change, followed by FSH and inhibin B. Measurements of E2 and FSH to know the progress of menopause have limitations, because they present great variability, and are modified by others Factors such as obesity and metabolic status. ${ }^{3}$ In fact, the change in the levels of FSH are less pronounced in obese women compared to non-obese women. Other peptides like as inhibin $\mathrm{A}^{4}$ follicular factor which together with estrogens may influence endocrine control, inhibiting to $\mathrm{FSH}$; therefore, the decrease of this factor is another indicator of follicular exhaustion. The FSH levels in women who already have menstrual irregularities fluctuate considerably, ${ }^{5}$ however, there is no marker predicting the last menstrual period (menopause transition). ${ }^{6}$

The AMH or Mullerian inhibitor (MIS) in women is expressed in granulosa cells. These belong to the transforming growth factor superfamily beta (TGF- $\beta$ ). AMH is a marker of prepuberal testicular function and an indicator of follicular reserve in adult women. ${ }^{7}$ Indeed, serum levels of AMH may represent the quantity and quality of the pool of ovarian follicles, as this is expressed during folliculogenesis, from the primary follicular phase to the antral phase. AMH appears to be the best marker reflecting decreased reproductive age. ${ }^{8}$ Moreover, $\mathrm{AMH}$ levels correlate with the number of antral follicles and age, and decrease with FSH levels. ${ }^{9}$ It has been suggested that AMH is independent of classical endocrine fluctuations of the menstrual cycle, without fluctuations such as FSH, luteinizing hormone (LH) and E2, which may be independent of the ovarian reserve. ${ }^{10}$

In addition to physical symptoms are also considered emotional symptoms. Since adolescence, women are at greater risk than men for depressive disorders. Traditionally, it is assumed that the transition to menopause or perimenopause is a period of greater depressive vulnerability, although there is some controversy. This period seems to be associated with the onset of depressive disorders, especially if there 
is a history of depression. The etiopathogenesis of perimenopause is explained by genetic, hormonal, psychological and socio cultural factors; However, no results are known to support any of these explanations. ${ }^{11}$

The above results in vasomotor modifications such as hot flashes whose pathophysiology is little known; however, it is known that the autonomic nervous system (ANS) performs an important yet undefined function. During an episode of embarrassment, symptoms of alteration are observed in autonomic control of the heart rate, which may participate in the mechanisms of regulation of the process. ${ }^{11}$

Hot flashes are the most common complaint that women go to for consultation; $32.9 \%$ of them present severe hot flashes, $43.1 \%$ present moderate and $20.4 \%$ mildly. ${ }^{12}$ These vasomotor symptoms modify the quality of life in women and have been reported to be more affected in those perimenopausal to postmenopausal. ${ }^{12}$ The maximum prevalence of hot flashes occurs one year after menopause, the average duration is 10.2 years; but the duration is still unclear. It has been observed that the onset of menopause predicts the duration of hot flashes. ${ }^{13}$

Eighty percent of menopausal women have hot flashes and night sweats, of which $9 \%$ have severe symptoms that affect their quality of life. Ideally, treatment options should be tailored to each woman, since, for most, vasomotor symptoms (VSM) resolve spontaneously in 3 to 5 years. ${ }^{14}$

Brown et al. ${ }^{15}$ found that systolic blood pressure increased after a hot flash, but it is not clear whether there is a causal relationship. This suggests the presence of a common mechanism in the central nervous system (CNS) for the onset of hot flashes and the systemic vasopressor response, and that both have psychological and physiological components. ${ }^{15}$

It is proposed that SVM are caused by the increase of endorphins in the hypothalamus in response to decreased estrogen levels, which alters the activity of neurotransmitters, especially in the serotonergic and noradrenergic pathways and blocks the thermoregulatory center which responds as an inappropriate mechanism of heat loss. ${ }^{16}$

The body thermoregulatory control has a lower and a higher limit. If the temperature range falls below the value, the mechanism is vasoconstriction, when the upper limit is exceeded, the mechanism is vasodilatation with production of moisture, sweating and heat loss through the skin. ${ }^{17}$ In humans, sympathetic innervation, a reflex of the cutaneous circulation, has two branches: a sympathetic noradrenergic vasoconstrictor system and an active non-noradrenergic vasodilator system. Vasoconstricting nerves respond to the release of nor adrenaline and co-transmitters including NPY which decreases blood flow to the skin. The vasodilator system has no rest tone and is only active during increases in body temperature, caused by exposure to heat or exercise. Cutaneous vasodilation is produced by cholinergic nerve transmitters, and nitric oxide, vasoactive intestinal peptide, prostaglandins, and substance $\mathrm{P}$ (neurokinin-1 receptors) have been proposed. It is interesting that there is no single mediator in cutaneous vasodilation. ${ }^{18-20}$

NPY affects vasoconstriction by the potential activation of the alpha adrenergic receptor. It is clear that norepinephrine and NPY act together to reduce blood flow to the skin during hypothermia, although NPY function is not known when it directly activates the cutaneous smooth vasculature or the noradrenergic mechanism. ${ }^{21}$ Moreover, NPY reduces the activity of the sympathetic efferent nerve pathways that reach brown adipose tissue and decrease heat dissipation. This peptide has multiple effects on energy metabolism, reproduction, neurogenesis and emotion. The vasoactive effects of NPY are known to be limited to long periods of stress. The involvement of NPY in basal vasomotor and sympathetic tone of in vivo vasoconstriction has been reported in both skeletal muscle and cutaneous circulation. ${ }^{22}$

NPY is a peptide with 36 amino acid residues and is widely distributed. In the female reproductive system regulates blood flow and contractile activity of vascular smooth muscle cells of the uterus, fallopian tubes, and secretory function of the ovary. The levels of NPY and its influence on blood flow in the female reproductive organs are related by fluctuations in the levels of ovarian steroid hormones $(\mathrm{OSH}){ }^{23}$ Moreover, NPY concentrations in obese and thin postmenopausal women are higher in comparison to young women, which suggests that it may be related to body mass index (BMI), and not only for differences in $\mathrm{OSH} .{ }^{24}$ The relationship between NPY and SVM in postmenopausal women wasreported. ${ }^{25}$ Therefore, the objective of this study is to identify the association between concentrations of anti-Müllerian hormone, neuropeptide Y, follicle stimulating hormone and estrogens with vasomotor symptoms in peri- and postmenopausal women. In addition, to find biological and psychological factors that allows the development of strategies to improve the quality of life in this period, because women live the last part of their lives at this stage.

\section{Methods}

\section{Study participants}

A non-probabilistic cross-sectional study was performed in consecutive cases in healthy peri and postmenopausal women attending Health Centers in Leon, Guanajuato. Based on previous studies, where there was an association between the hormonal concentrations described, a sample size of 110 women ${ }^{13}$ was considered for this study.

Healthy women aged 40 to 60 years without evidence of chronic or degenerative diseases were included. They were classified into two groups: group 1, perimenopausal women, those who presented menstrual irregularities with nine or fewer menstrual cycles in 12 months and group 2, postmenopausal women: who presented 12 consecutive months of amenorrhea. Women without hysterectomy or who have taken hormone replacement therapy less than or equal to six months were not included. Also, those who did not complete the records of hot flashes, pregnant, lactating, hysterectomy, chemotherapy, radiotherapy or hormone replacement therapy. ${ }^{26}$

This study did not represent any higher than minimum risk for women volunteers. The confidentiality of each person's data was guaranteed. The nature of the study, possible risks and benefits were explained and the informed consent letter of the participating women was obtained. This research gathered the principles described in the Helsinki Declaration and the General Health Law in the research section with a minimum risk classification. It was approved by the Ethics Committee of the Department of Medical Sciences of the University of Guanajuato.

The levels of NPY, FSH, E2 and AMH in the blood in fasting of all women were determined. In addition, a validated questionnaire (used in previous studies) was applied to request information about vasomotor symptoms and to determine the hot flashes at the beginning and at the end of the study. Subsequently, they were given a registry to describe the symptoms of the hot flashes and the evaluation was 
repeated at two weeks.

The questionnaire described schooling (years), marital status, weight, height, BMI, lifestyles: physical exercise (aerobic, anaerobic or mixed) evaluated on days per week, smoking (yes or not), consumption of alcoholic beverages (yes or not), gyneco-obstetric history: age of menarche, regularity of menstrual cycles, length of menstrual cycle, type of bleeding (qualitative assessment: mild, moderate and abundant), date of last menstruation (years of amenorrhea), number of pregnancies, births, number of cesareans, months of lactation. In addition, symptoms for depression were assessed, according to the Hamilton Belch Raphaelsen questionnaire ${ }^{27}$ with scores ranging from 0 to 39 ( 0 to 17: no depression, 18 to 25 : minor depression, and equal to or greater than 26: major depression); which includes depression, interest and vitality in work and occupation, feeling of fatigue, headaches, feelings of guilt, decreased verbal activity (are you tired of talking? or talking to you?), thoughts of suicide, slow thinking to speak, difficulty to focus, trouble falling asleep, trouble falling asleep at midnight, sleep disturbance in the morning, loss of sexual interest; while the anxiety scale was 0 to 19 , chest pain, dizziness palpitations, sweating, fears, tingling in hands and feet, empty nest syndrome (on a scale of 0 to 11), with questions such as: have lost the support of your family or husband?, do you feel unsatisfied with your family?, are you afraid of the family economy?, do you feel that you live without illusions?, do you consider that separation of children damages the stability of the family?. The perception of vasomotor symptoms was classified as mild, moderate and severe, the frequency of hot flashes was 2 to 5 hours, every 6 to 11 hours, every 12 to 23 hours, 1 to 2 days per week, 3 to 4 days per week, 5 to 6 days per week, 2 to 3 days per month, one day per month, less than one day per month, never and do not know. The duration of the evaluation was per minute or greater than one minute.

A $10 \mathrm{~mL}$ sample of peripheral venous blood in fasting was obtained, from which the serum was obtained, aliquoted and stored at $-70^{\circ} \mathrm{C}$ until use. Blood glucose and lipid profile was measurement. The

Table I General characteristics of peri- and postmenopausal women levels of NPY were determinate by radioimmunoassay following the manufacturer's instructions (RIA; KIT RK-049-03 por Internacional de Energía Nuclear, S.A de C.V.). The levels of $\mathrm{E}_{2}$ and FSH by Enzyme-Linked Immuno Sorbent Assay (EL10013 ANOGEN/ Follicle Stimulating Hormone (FSH) ELISA Kit, human), as well as LH (EL10011 ANOGEN/Luteinizing Hormone (LH) ELISA Kit, human) and AMH (KT-50091-S KAMIYA/Anti-Mullerian Hormone ELISA Kit (AMH), human) following the manufacturer's instructions.

\section{Statistical analysis}

The data were analyzed with descriptive statistics, with the statistical package Statistic (version 7). The Kolmogorov-Smirnov test and the homogeneity test of variances to determine the normality of the data were performed. The difference in symptoms, clinical parameters and hormonal concentrations in the peri- and postmenopause group were compared using the Student $\mathrm{t}$-test of independent groups for continuous variables, and the $\chi^{2}$ test or the Mann-Whitney U test for the discrete variables. The multiple regression technique was used to compare the factors associated with the symptoms, the SVM was considered as a dependent variable and as regressors age, schooling, BMI, parity, amenorrhea and FSH levels, E2, The NPY and the AMH. A P $<0.05$ was considered as significant difference.

\section{Results}

\section{General characteristics}

The total sample was 108 women, 57\% were perimenopausal (group 1) and $42 \%$ were postmenopausal (group 2). The age of the perimenopausal women was $45.8 \pm 3.1$ years and in the postmenopausal women $51.6 \pm 3.9$ years. The general characteristics of both populations are shown in (Table 1) (Table 2).

NPY and FSH levels were higher in postmenopausal women than in perimenopausal women ( $\mathrm{P}<0.3$ and $\mathrm{P}<0.01$, respectively). The concentrations of AMH and E2 were higher $(\mathrm{P}<0.001$ and $\mathrm{P}<0.001$, respectively) in the perimenopausal group (Table 3 ).

\begin{tabular}{|c|c|c|c|c|}
\hline & Group 1 & Group 2 & & \\
\hline Variable & $\begin{array}{l}\text { Perimenopausal* } \\
\mathrm{n}=61 \\
\dot{\mathrm{X}} \pm \mathrm{SD}\end{array}$ & $\begin{array}{l}\text { Postmenopausal* } \\
\mathrm{n}=47 \\
\dot{\mathrm{X}} \pm \text { SD }\end{array}$ & $\mathbf{t}$ & $\mathbf{P}$ \\
\hline Body weight & $57.4 \pm 26.6$ & $69.5 \pm 12.5$ & -2.87 & 0.004 \\
\hline Height (m) & $1.52 \pm 0.0$ & $1.55 \pm 0.0$ & 1.3 & 0.19 \\
\hline BMI $\left(\mathrm{kg} / \mathrm{cm}^{2}\right)$ & $29.2 \pm 4.7$ & $29.1 \pm 5.5$ & 0.12 & 0.9 \\
\hline Glucose (mg/dL) & $92.1 \pm 11.2$ & $92.6 \pm 12.4$ & -0.24 & 0.8 \\
\hline Triglyceride (mg/dL) & $149 \pm 60.5$ & $150.3 \pm 57.6$ & -0.1 & 0.92 \\
\hline Total cholesterol (mg/dL) & $177 \pm 35.8$ & $193.2 \pm 29.3$ & -2.59 & 0.01 \\
\hline $\mathrm{HDL}(\mathrm{mg} / \mathrm{dL})$ & $51.1 \pm 8.1$ & $50.79 \pm 7.3$ & 0.18 & 0.85 \\
\hline $\mathrm{LDL}(\mathrm{mg} / \mathrm{dL})$ & $95.7 \pm 32.7$ & $111.81 \pm 27.9$ & -2.71 & 0.008 \\
\hline Scholarship (years) & $6.97 \pm 4.3$ & $5.62 \pm 3.7$ & 1.69 & 0.09 \\
\hline Exercise (per week) & $0.51 \pm 0.7$ & $0.49 \pm 0.8$ & 0.12 & 0.9 \\
\hline Menarche (years) & $12.2 \pm 1.9$ & $12.72 \pm 1.6$ & -1.34 & 0.18 \\
\hline $\begin{array}{l}\text { Regularity of the menstrual cycle } \\
\text { (days) }\end{array}$ & $0.78 \pm 0.4$ & $0.87 \pm 0.3$ & -1.92 & 0.05 \\
\hline Length of menstrual cycle (d) & $21.6 \pm 12.5$ & $25.70 \pm 9.2$ & -1.9 & 0.05 \\
\hline
\end{tabular}


Table Continued

\begin{tabular}{|c|c|c|c|c|}
\hline \multirow{3}{*}{ Variable } & Group 1 & Group 2 & \multirow{3}{*}{ t } & \multirow{3}{*}{$\mathbf{P}$} \\
\hline & $\begin{array}{l}\text { Perimenopausal* } \\
n=61\end{array}$ & $\begin{array}{l}\text { Postmenopausal* } \\
n=47\end{array}$ & & \\
\hline & $\dot{\mathbf{X}} \pm \mathbf{S D}$ & $\dot{\mathbf{X}} \pm \mathrm{SD}$ & & \\
\hline Pregnancies & $5.0 \pm 3.0$ & $6.4 \pm 3.3$ & -2.44 & 0.016 \\
\hline Birth & $3.4 \pm 3.1$ & $5.1 \pm 3.4$ & -2.67 & 0.009 \\
\hline Abortions & $0.6 \pm 1.0$ & $0.7 \pm 1.5$ & -0.6 & 0.54 \\
\hline Cesareans & $0.9 \pm 1.2$ & $0.6 \pm 0.9$ & 1.61 & 0.11 \\
\hline Breastfeeding (months) & $0.92 \pm 0.2$ & $0.8 \pm 0.3$ & 1.36 & 0.17 \\
\hline $\begin{array}{l}\text { Frequency of hot flashes (day/week/ } \\
\text { month) }\end{array}$ & $4.2 \pm 2.3$ & $4.3 \pm 2.4$ & -0.21 & 0.83 \\
\hline Duration of hot flashes (min) & $6.5 \pm 2.5$ & $6.6 \pm 2.5$ & -0.19 & 0.84 \\
\hline
\end{tabular}

BMI, body mass index

*Data expressed as mean \pm standard deviation. Student's T-test, $\mathrm{P}<0.05$.

Table 2 Symptoms of menopause: comparison between peri- and postmenopausal women

\begin{tabular}{|c|c|c|c|c|}
\hline \multirow{3}{*}{ Variable } & Group 1 & Group 2 & \multirow{3}{*}{$\mathbf{t}$} & \multirow{3}{*}{$\mathbf{P}$} \\
\hline & $\begin{array}{l}\text { Perimenopausal* } \\
n=61\end{array}$ & $\begin{array}{l}\text { Postmenopausal* } \\
n=47\end{array}$ & & \\
\hline & $\dot{\mathrm{X}} \pm \mathrm{SD}$ & $\dot{\mathbf{X}} \pm$ SD & & \\
\hline Depression & $9.4 \pm 4.5$ & $8.36 \pm 4.3$ & 1.27 & 0.2 \\
\hline Sleeping problems & $2.9 \pm 1.9$ & $2.96 \pm 1.9$ & 0.03 & 0.97 \\
\hline NSS & $2.5 \pm 1.5$ & $2.38 \pm 1.4$ & 0.66 & 0.51 \\
\hline Anxiety & $10.8 \pm 5.2$ & $9.49 \pm 4.5$ & 1.39 & 0.16 \\
\hline ENS & $4.8 \pm 3.0$ & $4.51 \pm 3.2$ & 0.51 & 0.6 \\
\hline Vaginal dryness & $0.6 \pm 0.5$ & $0.57 \pm 0.5$ & 0.33 & 0.73 \\
\hline Dyspareunia & $0.4 \pm 0.5$ & $0.38 \pm 0.5$ & 0.28 & 0.77 \\
\hline Hot flashes & $0.5 \pm 0.5$ & $0.89 \pm 0.3$ & -0.85 & 0.0002 \\
\hline Intensity of hot flashes & $1.6 \pm 0.5$ & $1.67 \pm 0.5$ & -0.62 & 0.53 \\
\hline Number of hot flashes & $37.5 \pm 39.2$ & $55.6 \pm 50.3$ & -2.1 & 0.037 \\
\hline
\end{tabular}

NSS, non-specific symptoms; ENS, empty nest syndrome

*Data expressed as mean \pm standard deviation. Student's T-test, $\mathrm{P}<0.05$.

Table 3 Comparison of hormonal concentrations in both groups of women

\begin{tabular}{|c|c|c|c|c|c|c|}
\hline \multirow{3}{*}{ Hormones } & \multicolumn{2}{|l|}{ Group I } & \multicolumn{4}{|l|}{ Group 2} \\
\hline & $\begin{array}{l}\text { Perimenopausal } \\
(n=6 I)\end{array}$ & Percentile (25 to 75$)$ & $\begin{array}{l}\text { Postmenopausal } \\
(n=49)\end{array}$ & $\begin{array}{l}\text { Percentile ( } 25 \\
\text { to } 75)\end{array}$ & $\mathbf{z}$ & $\mathbf{P}$ \\
\hline & Median & & Median & & & \\
\hline NPY (pg/mL) & 20.7 & 10 to 43.2 & 29.1 & 10 to 43.2 & -1.00 & 0.315 \\
\hline AMH (ng/mL) & 8.5 & 4.1 to 15.5 & 4.9 & 4.1 a 15.5 & 2.46 & 0.013 \\
\hline E2 (pg/mL) & 8.4 & I.5 to 19.3 & 0.5 & 1.52 to 19.3 & 4.32 & 0.001 \\
\hline $\mathrm{FSH}(\mathrm{mUl} / \mathrm{mL})$ & 34.6 & 14.0 to 67.8 & 59.9 & 14.0 to 67.8 & -2.32 & 0.019 \\
\hline
\end{tabular}

NPY, neuropeptide Y;AMH, anti-müllerian hormone; E2, estradiol; FSH, follicle stimulating hormone Mann-Whitney U test was performed. 


\section{Analysis of multiple linear regression and variables associated with vasomotor symptoms in both groups of women}

In Table 4, the analysis of multiple linear regression and the variables associated with hot flash described. Sleep problems, FSH concentration and BMI (adjusted $\mathrm{R}^{2}=0.13, \mathrm{P}<0.006, \mathrm{P}<0.005$ and $\mathrm{P}<0.04$, respectively) are independent predictors of the duration of hot flashes (Model 1). The frequency of hot flushes correlated negatively with FSH and anxiety (adjusted $\mathrm{R}^{2}=0.070, \mathrm{P}<0.006$ and $\mathrm{P}$ Table 4 Multiple regressions in peri- and postmenopausal women
$<0.0390$, respectively, Model 2). NPY concentrations were associated with high density lipoprotein (HDL) levels, schooling results and number of births (adjusted $\mathrm{R}^{2}=0.1, \mathrm{P}<0.013, \mathrm{P}<0.007$ and $\mathrm{P}<0.03$, respectively). HDL was an independent predictor of AMH (adjusted $\mathrm{R}^{2}=0.03, \mathrm{P}<0.04$, Model 4).

The two-week record of hot flashes in both groups of women correlated positively with FSH concentration (adjusted $\mathrm{R}^{2}=0.050, \mathrm{P}$ $<0.02$; Model 5). In the latter model all hormonal levels were added, and no differences were found ( $\mathrm{P}>0.05)$ except for FSH levels.

\begin{tabular}{|c|c|c|c|c|c|}
\hline Dependent variable & Regressor & Beta \pm SD Beta & B \pm SD B & $\mathbf{t}$ & $\mathbf{P}$ \\
\hline \multicolumn{6}{|c|}{ Duration_Hot flushes in peri- and postmenopausal women. (Model I).Adjusted $R^{2}=0.13806210, F(3,106)=6.8197, P<0.00030$. } \\
\hline & Intercept & & $2.05 \pm 1.39$ & 1.48 & 0.14 \\
\hline & Slepping problems & $0.25 \pm 0.09$ & $0.32 \pm 0.12$ & 2.79 & 0.006 \\
\hline & $\mathrm{FSH}$ & $0.26 \pm 0.09$ & $0.02 \pm 0.01$ & 2.86 & 0.005 \\
\hline & BMI & $0.19 \pm 0.09$ & $0.09 \pm 0.04$ & 2.09 & 0.04 \\
\hline \multicolumn{6}{|c|}{ Frecuency_Hot flushes in peri- and postmenopausal women (Model 2). Adjusted $R^{2}=0.07291290, F(2,107)=5.2863, P<0.00647$. } \\
\hline & Intercept & & 0.577 & 10.34 & 0 \\
\hline & $\mathrm{FSH}$ & $-0.23 \pm 0.09$ & $-0.014 \pm 0.00$ & -2.52 & 0.006 \\
\hline & Anxiety & $-0.19 \pm 0.09$ & $-0.09 \pm 0.04$ & -2.09 & 0.04 \\
\hline \multicolumn{6}{|c|}{ NPY $(\mathrm{pg} / \mathrm{mL})$ in peri- and postmenopausal women (Model 3). Adjusted $\mathrm{R}^{2}=0.12263959, \mathrm{~F}(3,106)=6.0788, \mathrm{P}<0.00074$. } \\
\hline & Intercept & & $-88.32 \pm 116.04$ & -0.76 & 0.448 \\
\hline & HDL & $0.23 \pm 0.09$ & $5.12 \pm 2.02$ & 2.54 & 0.013 \\
\hline & Scolarship & $-0.26 \pm 0.09$ & $-11.16 \pm 4.02$ & -2.78 & 0.007 \\
\hline & Birth & $-0.21 \pm 0.09$ & $-10.95 \pm 5.03$ & -2.18 & 0.032 \\
\hline \multicolumn{6}{|c|}{ AMH $\left(\mathrm{ng} / \mathrm{mL}\right.$ ) in peri- and postmenopausal women (Model 4). Adjusted $\mathrm{R}^{2}=0.02924307, \mathrm{~F}(\mathrm{I}, \mathrm{I08})=4.2835, \mathrm{P}<0.04087$. } \\
\hline & Intercept & & $32.07 \pm 10.02$ & 3.19 & 0.001 \\
\hline & HDL & $-0.19 \pm 0.09$ & $5.12 \pm 2.02$ & -2.07 & 0.04 \\
\hline \multicolumn{6}{|c|}{ Registry_2weeks_Hot flushes peri- and postmenopausal (Model 5).Adjusted $R^{2}=0.05027 \mid 33, F(I, I 06)=6.6638, P<0.0 I I 2 I$. } \\
\hline & Intercept & & $30.83 \pm 7.04$ & 4.37 & 0 \\
\hline & $\mathrm{FSH}(\mathrm{mUl} / \mathrm{mL})$ & $0.24 \pm 0.09$ & $0.27 \pm 0.107$ & 2.58 & 0.01 \\
\hline
\end{tabular}

\section{Discussion}

The mechanism that causes vasomotor symptoms (VMS) is not completely understood. It has been suggested that the reduction in estrogen concentrations leads to a decrease in endorphin concentrations in the hypothalamus and increases the release of serotonin and norepinephrine, which causes a decrease in neurotransmitters and "misalign" the thermoregulatory center. ${ }^{28,29}$

There are few studies that report the association between the frequency and duration of VSM with hormone levels. The present study revealed the association of hot flashes with sleep problems. Women with a high frequency of hot flashes have more disturbed sleep. ${ }^{30}$ Women with moderate to severe hot flashes are associated with insomnia, fragmented sleep, and altered sleep architecture. ${ }^{31}$ Individuals with insomnia are likely to focus during the day exaggerated on potential consequences, the result of this autonomic and emotional activation approach result in problems, which worsens the disturbance, in chronic sleep disorder processes. ${ }^{32}$

Women who have hot flashes may engage in maladaptive cognitions and reduce the perception of tranquility. Night sweats and hot flashes associated with poor sleep quality suggest that the hormonal environment is a critical variable in predicting sleep disruption. ${ }^{33}$ The mechanisms by which steroid hormones influence sleep have been poorly studied, with a possible contribution of gonadotropins in the regulation of CNS activities. The release of LH has been positively associated with the frequency and severity of hot flashes, even in the presence of relatively high concentrations of estrogen.

Although the pulses of LH are not the immediate cause of hot flashes, the release of LH occurs with the onset and increase of core body temperature. ${ }^{34}$ It is not known whether the pulsatile activity of LH is associated with acute changes in body temperature, which disrupt sleep, or if the release of gonadotropins has long-term effects on body temperature. Also, levels of FSH and $\mathrm{E}_{2}$ can influence the onset and exacerbation of VMS. ${ }^{35}$ 
In the present study, the duration of hot flashes was found to be associated with BMI. The mechanism by which BMI increases the chances of hot flashes is unclear. One of the possible explanations between measures of body fat ( $\%$ body fat) and circulating concentrations of leptin in adults is that it mediates the formation of circulating estrogens androgen precursors and affects thermoregulation. Leptin acts on specific receptors in the hypothalamus, a region associated with energy expenditure. ${ }^{36}$

Adipose tissue has endocrine and thermoregulatory properties; however, its thermoregulatory function is observed more in relation to VMS. Adiposity could inhibit heat dissipation; therefore, more vasomotor events may be necessary to dissipate a greater amount of heat required. Adipose tissue has the strongest insulating ability of any body tissue, and the decrease in the ability of obese people to effectively dissipate heat is well documented. ${ }^{37}$

In the present study, a positive association was found between NPY and HDL levels. The results suggest that NPY produced in the hypothalamus influences appetite. The NPY Y1 receptor is highly expressed in human adipocytes, which inhibits lipolysis. ${ }^{38}$ NPY stimulates lipoprotein lipase (LPL), the enzyme that catalyzes the uptake of triglycerides (TG) in cells, by generating free fatty acids and b-monoacylglycerol from lipoprotein complexes. ${ }^{39}$ As there is no minimum of de novo lipogenesis in adipose tissue in humans, the re-esterification of free fatty acids to glycerol is the main source of accumulated lipids. It's probably that in humans, NPY has an important role in lipid metabolism. LPL is also involved in the metabolism of lipoproteins. Low LPL activity delays the elimination of TG-rich particles, resulting in high amounts of TG and a decrease in HDL. Conversely, when LPL activity is increased (as with NPY stimulation), very low density lipoprotein (VLDL) and chylomicrons are eliminated from the circulation and, therefore, HDL levels increase.

Thus, stimulation of NPY leads to increased LPL activity in white fat and decreased thermogenesis of brown fat.$^{38}$ There is evidence of association of single nucleotide polymorphism (SNPs) with NPY5R gene and dyslipidemia (high concentrations of TG and reduction of HDL) in the Mexican population. ${ }^{40}$

In addition, we found the NPY association with schooling and number of births. Patients who presented higher levels of NPY resulted in a lower level of schooling and fewer births. The results suggest the possibility that stress affects women who have low education. Early adverse events in life as environmental influences have an effect on long-term development in the regulation of the hypothalamic-pituitaryadrenal (HPA) axis. The interaction between NPY gene variation and adverse family environment influences individual susceptibility to the persistence of neuroendocrine changes.

Long-lasting changes in the regulation of HPA axis activity could have an impact on different brain systems, especially in the hippocampus: in the remodeling of hippocampal formation by repeated stress, which includes loss of spinal synapses, shortening of dendrites and suppression of neurogenesis, which impede termination of the hippocampus-induced stress response, leading to high HPA axis activity. A SNP polymorphism in the NPY gene promoter (rs16147) has been reported to be associated with psychosocial adversity, which modifies the ACTH and cortisol response to acute psychosocial stress; therefore, it may influence the expression of the NPY gene. This could moderate the impact of psychosocial adversity and vulnerability to stress. ${ }^{41}$
Finally, we demonstrate an association of AMH with lower levels of HDL. The mechanism of dietary effect in total follicles remains undetermined. ${ }^{42}$ Increased concentrations of AMH favor the release of gonadotrophins in polycystic ovary syndrome (PCOS). This increase can be used as a new surrogate marker of ovarian hyperandrogenism in patients with PCOS, and also as a new cardiovascular risk factor. ${ }^{43}$

\section{Conclusion}

Women in postmenopause have more hot flashes compared to women in perimenopause. Twenty-two point seven percent of all women perceive hot flashes as moderate, followed by mild and severe hot flashes. There are differences in levels of the anti-Müllerian hormone, estradiol and follicle stimulating hormone in both groups. In addition, there are associations of duration of hot flashes with sleep problems, follicle stimulating hormone and body mass index. The frequency of hot flashes correlated with follicle stimulating hormone and anxiety.

\section{Acknowledgements}

The authors thank the economic financing with the project CONACYT No. 32285

\section{Conflict of interest}

The authors declare that they have no conflict of interest.

\section{References}

1. Practice Committee of American Society for Reproductive Medicine. The menopausal transition. Fertil Steril. 2008;90(5 Suppl):S61-5.

2. Shulman LP. Androgens and menopause. Minerva Ginecol. 2009;61(6):491-7.

3. Su HI, Freeman EW. Hormone changes associated with the menopausal transition. Minerva Ginecol. 2009;61(6):483-9.

4. Randolph JF, Zheng H, Sowers MR. Change in follicle-stimulating hormone and estradiol across the menopausal transition: effect of age at the final menstrual period. J Clin Endocrinol Metab. 2011;96(03):74654.

5. Farage MA, Neill S, MacLean AB. Physiological changes associated with the menstrual cycle: a review. Obstet Gynecol Surv. 2009;64(1):5872 .

6. Syed Alwi SA, Lee PY, Awi I, et al. The menopausal experience among indigenous women of Sarawak, Malaysia. Climacteric. 2009;12(6):548-56.

7. Josso N, Picard JY, Rey R, et al. Testicular anti-Müllerian hormone: history, genetics, regulation and clinical applications. Pediatr Endocrinol Rev. 2006;3(4):347-58.

8. La Marca A, Volpe A. Anti-Müllerian hormone (AMH) in female reproduction: is measurement of circulating AMH a useful tool? Clin Endocrinol (Oxf). 2006;64(6):603-10.

9. de Vet A, Laven JS, de Jong FH, et al. Antimüllerian hormone serum levels: a putative marker for ovarian aging. Fertil Steril. 2002; 77(2):357-62.

10. Hehenkamp WJ, Looman CW, Themmen AP. Anti-Müllerian hormone levels in the spontaneous menstrual cycle do not show substantial fluctuation. J Clin Endocrinol Metab. 2006;91(10):4057-63.

11. Gerber LM, Sievert LL, Warren K, et al. Hot flashes are associated with increased ambulatory systolic blood pressure. Menopause. 
2007;14(2):308-15.

12. Ayranci U, Orsal O, Orsal O, et al. Menopause status and attitudes in a Turkish midlife female population: an epidemiological study. $B M C$ Womens Health. 2010;10:1.

13. Freeman EW, Sammel MD, Lin H, et al. Duration of Menopausal Hot Flushes and Associated Risk Factors. Obstet Gynecol. 2011;117(5):1095-104.

14. Shen W, Stearns V. Treatment strategies for hot flushes. Expert Opin Pharmacother. 2009;10(7):1133-44.

15. Brown DE, Sievert LL, Morrison LA, et al. Relationship between ho flashes and ambulatory blood pressure: the Hilo women's health study. Psychosom Med. 2011;73(2):166-72.

16. Nelson HD, Vesco KK, Haney E, et al. Nonhormonal therapies for menopausal hot flashes: systematic review and meta-analysis. JAMA. 2006;295(17):2057-71.

17. Rossmanith WG, Ruebberdt W. What causes hot flushes? The neuroendocrine origin of vasomotor symptoms in the menopause. Gynecol Endocrinol. 2009;25(5):303-14.

18. Charkoudian N. Mechanisms and modifiers of reflex induced cutaneous vasodilation and vasoconstriction in humans. J Appl Physiol (1985). 2010;109(4):1221-28.

19. Kellogg DL. In vivo mechanisms of cutaneous vasodilation and vasoconstriction in humans during thermoregulatory challenges. $J \mathrm{Appl}$ Physiol (1985). 2006;100(5):1709-18.

20. Freedman RR, Woodward S, Mayes MM, Woodward S, Mayes MM. Nonneural mediation of digital vasodilation during menopausal hot flushes. Gynecol Obstet Invest. 1994;38(3):206-9.

21. Charkoudian N. Skin blood flow in adult human thermoregulation: how it works, when it does not, and why. Mayo Clin Proc. 2003;78(5):60312.

22. Hodges GJ, Jackson DN, Mattar L, et al. Neuropeptide Y and neurovascular control in skeletal muscle and skin. Am J Physiol Regul Integr Comp Physiol. 2009;297(3):546-55.

23. Markiewicz W, Jaroszewski JJ, Bossowska A, et al. NPY: its occurrence and relevance in the female reproductive system. Folia Histochem Cytobiol. 2003;41(4):183-92.

24. Baranowska B, Radzikowska M, Wasilewska-Dziubínska E, et al. Relationship among leptin, neuropeptide Y, and galanin in young women and in postmenopausal women. Menopause. 2000;7(3):149-55.

25. Wyon YA, Spetz AC, Theodorsson GE, et al. Concentrations of calcitonin gene-related peptide and neuropeptide $\mathrm{Y}$ in plasma increase during flushes in postmenopausal women. Menopause. 2000;7(1):2530 .

26. Iñigo Riesgo CA, Torres Gómez LG, Lofte Navarro CA, et al Cardiovascular risk factors in climacteric. Ginecol Obstet Mex. 2009;77(12):535-43

27. García-Campos R, Aguilar-Zavala H, Malacara JM. Symptoms at menopause and care of grandchildren. Climacteric. 2010;13(5):492-8.

28. Gallicchio L, Miller SR, Zacur H, et al. Flaws. Hot flashes and blood pressure in midlife women. Maturitas. 2010;65(1):69-74.

29. Heidi D Nelson. Menopause. Lancet. 2008;371(9614):760-70.

30. Schilling C, Gallicchio L, Miller SR, et al. Relation of bodymass and sex steroid hormone levels to hot flushes in a sample of mid-life women. Climacteric. 2007;10(1):27-37.

31. Brown JP, Gallicchio L, Flaws JA, et al. Relations among menopausal symptoms, sleep disturbance and depressive symptoms in midlife. Maturitas. 2009;62(2):184-9.

32. Ensrud KE, Stone KL, Blackwell TL, et al. Frequency and severity of hot flashes and sleep disturbance in postmenopausal women with hot flashes. Menopause. 2009;16(2):286-92.

33. Sateia MJ, Nowell PD. Insomnia. Lancet. 2004;364(9449):1959-73.

34. Kloss JD, Tweedy K, Gilrain K. Psychological factors associated with sleep disturbance among perimenopausal women. Behav Sleep Med. 2004;2(4):177-90.

35. Murphy PJ, Campbell SS. Sex hormones, sleep, and core body temperature in older postmenopausal women. Sleep. 2007;30(12):178894.

36. Sowers MF, Zheng H, Kravitz HM, et al. Sex steroid hormone profiles are related to sleep measures from polysomnography and the Pittsburgh Sleep Quality Index. Sleep. 2008;31(10):1339-49.

37. Alexander C, Cochran CJ, Gallicchio L, et al. Serum leptin levels, hormone levels, and hot flashes in midlife women. Fertil Steril. 2010;94(3):1037-43.

38. Thurston RC, Sowers MR, Chang Y, et al. Adiposity and reporting of vasomotor symptoms among midlife women: the study of women's health across the nation. Am J Epidemiol. 2008;167(1):78-85.

39. Blumenthal JB, Andersen RE, Mitchell BD, et al. Novel neuropeptide $\mathrm{Y} 1$ and $\mathrm{Y} 5$ receptor gene variants: associations with serum triglyceride and high-density lipoprotein cholesterol levels. Clin Genet. 2002;62(3):196-202.

40. Coletta DK, Schneider J, Stern MP, et al. Association of neuropeptide Y receptor Y5 polymorphisms with dyslipidemia in Mexican Americans. Obesity (Silver Spring). 2007;15(4):809-15.

41. Witt SH, Buchmann AF, Blomeyer D, et al. Interaction between a neuropeptide $\mathrm{Y}$ gene polymorphism and early adversity modulates endocrine stress responses. Psychoneuroendocrinology. 2011;36(7):1010-20.

42. Appt SE, Chen H, Goode AK, et al. The Effect of Diet and Cardiovascular Risk on Ovarian Aging in Cynomolgus Monkeys (Macacafascicularis). Menopause. 2010;17(4):741-748.

43. Skałba P, Cygal A, Madej P, et al. Is the plasma anti-Müllerianhormone (AMH) level associated with body weight and metabolic, and hormonal disturbances in women with and without polycystic ovary syndrome? Eur J Obstet Gynecol Reprod Biol. 2011;158(2):254-9. 